\title{
Guest editorial: special issue on "knowledge extraction from low quality data: theoretical, methodological and practical issues"
}

\author{
Luciano Sánchez • Inés Couso
}

Published online: 6 October 2011

(C) Springer-Verlag 2011

Knowledge extraction techniques depend on the quality of the available data. In the best case, the deviation between the perceived information and the actual values of the variables is small and can be safely ignored. On other occasions this difference is relevant but a probability distribution can characterize the observation error. In the less favorable scenario, to which we will refer as "low quality data", the observation error is relevant but it cannot be aptly characterized by a unique probability distribution. Data with missing features, censored or comprising nested confidence intervals are particular cases of this.

This special issue collects twelve papers about the extraction of knowledge from low quality data. On one hand, there are theoretical contributions, discussing different characterizations of the sources of uncertainty (possibility theory, interval data, fuzzy sets, imprecise probabilities, etc.). On the other hand, there are methodological and empirical studies, related to the design and validation of systems on the basis of incomplete and imprecise information, including algorithms for learning association rules, classification and regression models with uncertain attributes and/or labels.

The first paper, titled "Partially supervised independent factor analysis using soft labels elicited from multiple experts: application to railway track circuit diagnosis" describes a new noiseless independent factor analysis

\footnotetext{
L. Sánchez $(\bowtie)$

Computer Science Department, University of Oviedo,

Campus de Viesques, 33071 Gijón, Asturias, Spain

e-mail: luciano@uniovi.es

I. Couso

Statistics Department, University of Oviedo,

Facultad de Ciencias, 33071 Oviedo, Asturias, Spain

e-mail: couso@uniovi.es
}

model applied to the diagnosis of railway track circuits. Partial information from domain experts, expressed as belief functions, is combined with measurement data. The authors propose to handle the learning of this statistical model in a soft-supervised way using imperfect information on the system component states.

In the second paper, "Singular spectral analysis of illknown signals and its application to predictive maintenance of windmills with SCADA records" a possibilistic generalization of the singular spectral analysis is proposed. This method produces a list of fuzzy bounds of the energy of an ill-known signal at different frequencies. By comparing these bounds to the expected energy of colored noise, those parts of the historical records of the supervision system of the windmill that are relevant for the prediction of mechanical failures can be identified.

The third paper, "A fuzzy interval analysis approach to kriging with ill-known variogram and data", proposes an approach to handle epistemic uncertainty appearing in the kriging methodology (predicting a spatial phenomenon at non sampled locations from an estimated random function) when the collected data contains errors that can be modeled by intervals or fuzzy intervals, while at the same time the choice of parameter values for the theoretical variogram is not completely determined.

These first three papers model the non random (epistemic) uncertainty in the data by means of families of probabilities. However, a different theoretical framework is assumed in both the fourth and the fifth papers. The minimization of a crisp distance between fuzzy sets is the basis of the work, "A fuzzy regression model based on distances and random variables with crisp input and fuzzy output data: a case study in biomass production", where a new generalization of the least-squares technique is introduced for solving a regression problem where the independent 
variable is partially unknown and can be modeled by triangular fuzzy numbers. In a similar fashion, in the paper "A cooperative coevolutionary approach dealing with the skull-face overlay uncertainty in forensic identification by craniofacial superimposition" the different sources of uncertainty are coped with by landmarks with fuzzy coordinates, and a crisp distance between fuzzy sets is used, as before, for assessing the matching between a skull and a face. Genetic algorithms are used for optimizing this distance and thus for finding the best match in the skullface overlay process, the main task in craniofacial superimposition.

The next two papers are related to problems of signal processing under imperfect knowledge. The sixth paper "Towards interval-based non-additive deconvolution in signal processing" solves the problem of reconstructing a signal from its observations via a sensor device whose impulse response is only partially known. The relationship between the measurement and the signal is described by means of a concave capacity, and the convolution concept to a concave set of impulse responses. The reconstructed signal is interval-valued, reflecting the poor knowledge of the sensor impulse response. The seventh paper "Filtering with clouds" focuses in the problem of selecting a kernel to filter a given digital signal. The authors propose to model sets of kernels by clouds (convex sets of probabilities), obtaining an interval-valued filtered signal, as in the preceding paper.

The following three papers investigate different strategies for designing classifiers with uncertain data. The eighth paper, "A K-nearest neighbours method based on imprecise probabilities" analyzes the foundations (in terms of lower previsions) of the statistical classification with imprecise data. The ninth paper, "Extending of information processing in a fuzzy random forest ensemble" is too devoted to the study of classifiers with imprecise data; a new learning algorithm for obtaining ensembles of decision trees from imperfect data is proposed therein, along with experimental results for sets of data including missing values, stochastic uncertainty and fuzzy data. In the last place of this block, the particular case of low quality datasets having missing features is explored from a different perspective in the tenth paper, "Missing data imputation for fuzzy rule based classification systems", that contains an exhaustive study of many different imputation procedures, that are empirically studied in combination with fuzzy rule-based classification.

The last two papers are related to the learning of association rules from data with different kinds of uncertainty. The eleventh paper in this issue, "Mining fuzzy association rules from low quality data" proposes a new data-mining algorithm for extracting interesting knowledge from databases with imprecise data, integrating certain concepts about the representation of interval valued and fuzzy data with the fuzzy apriori mining algorithm. This algorithm has demonstrated its usefulness as a diagnostic tool for detecting dyslexia in early childhood. Lastly, the paper "New algorithms for finding approximate frequent item sets" presents two new algorithms for obtaining approximate, fault-tolerant or fuzzy item sets. These sets arise when the definition of support of an item in a transaction is relaxed and it is allowed that some items of a given set are missing from a transaction.

Finally, as guests editors of this special issue, we would like to thank all the authors for their contributions and the referees for their important cooperation.

Luciano Sánchez and Inés Couso 